\title{
Visual percepts evoked with an intracortical 96-channel microelectrode array inserted in human occipital cortex
}

\author{
Eduardo Fernández, ${ }^{1,2,3}$ Arantxa Alfaro, ${ }^{1,2,4}$ Cristina Soto-Sánchez,, ${ }^{1,2}$ Pablo Conzalez-Lopez, ${ }^{1,5}$ Antonio M. Lozano, ${ }^{1}$ \\ Sebastian Peña, ${ }^{1}$ Maria Dolores Grima, ${ }^{1}$ Alfonso Rodil, ${ }^{1}$ Bernardeta Gómez, ${ }^{1}$ Xing Chen, ${ }^{6}$ Pieter R. Roelfsema, ${ }^{6,7}$ John D. Rolston, ${ }^{8}$ \\ Tyler S. Davis, ${ }^{3,8}$ and Richard A. Normann ${ }^{3,8}$ \\ 'Bioengineering Institute and Cátedra Bidons Egara, University Miguel Hernández, Elche, Spain. ${ }^{2}$ CIBER Research Center on Bioengineering, Biomaterials and Nanomedicine (CIBER BBN), Madrid, \\ Spain. ${ }^{3}$ John Moran Eye Center and Biomedical Engineering, University of Utah, Salt Lake City, Utah, USA. ${ }^{4}$ Servicio de Neurologia, Hospital Vega Baja, Orihuela, Spain. ${ }^{5}$ Servicio de Neurocirugia, Hospital \\ Universitario de Alicante, Alicante, Spain. ${ }^{6}$ Department of Vision \& Cognition, Netherlands Institute for Neuroscience, Amsterdam, Netherlands. ${ }^{7}$ Department of Integrative Neurophysiology, Center for \\ Neurogenomics and Cognitive Resarch, VU University, and Department of Psychiatry, Academic Medical Center, Amsterdam, Netherlands. ${ }^{8}$ Departments of Neurosurgery and Biomedical Engineering, \\ University of Utah, Salt Lake City, Utah, USA.
}

BACKCROUND. A long-held goal of vision therapy is to transfer information directly to the visual cortex of blind individuals, thereby restoring a rudimentary form of sight. However, no clinically available cortical visual prosthesis yet exists.

METHODS. We implanted an intracortical microelectrode array consisting of 96 electrodes in the visual cortex of a 57-year-old person with complete blindness for a 6-month period. We measured thresholds and the characteristics of the visual percepts elicited by intracortical microstimulation.

RESULTS. Implantation and subsequent explantation of intracortical microelectrodes were carried out without complications. The mean stimulation threshold for single electrodes was $66.8 \pm 36.5 \mu \mathrm{A}$. We consistently obtained high-quality recordings from visually deprived neurons and the stimulation parameters remained stable over time. Simultaneous stimulation via multiple electrodes was associated with a significant reduction in thresholds $(P<0.001$, ANOVA) and evoked discriminable phosphene percepts, allowing the blind participant to identify some letters and recognize object boundaries.

CONCLUSIONS. Our results demonstrate the safety and efficacy of chronic intracortical microstimulation via a large number of electrodes in human visual cortex, showing its high potential for restoring functional vision in the blind.

TRIAL REGISTRATION. ClinicalTrials.gov identifier NCT02983370.

FUNDING. The Spanish Ministerio de Ciencia Innovación y Universidades, the Generalitat Valenciana (Spain), the Europan Union's Horizon 2020 programme, the Bidons Egara Research Chair of the University Miguel Hernández (Spain), and the John Moran Eye Center of the University of Utah.

\section{Introduction}

The artificial restoration of vision in blind persons by direct electrical stimulation of the visual cortex has been a subject of study since the early work of Brindley (1-3) and Dobelle and Mladjilovski (4-6). They showed that visual percepts (phosphenes) could be evoked by electrical stimulation of the visual cortex via an array of electrodes implanted on the surface of a subject's visual cortex. More recently, Beauchamp et al. (7) implanted a new visual cortical prosthetic device with 60 surface electrodes, the Orion

\section{Delated Commentary: https://doi.org/10.1172/JCl154983}

Conflict of interest: PRR and XC are cofounders and shareholders of a neurotechnology startup, Phosphoenix (Netherlands).

Copyright: @ 2021, American Society for Clinical Investigation.

Submitted: May 11, 2021; Accepted: September 28, 2021; Published: December 1, 2021. Reference information: J Clin Invest. 2021;131(23):e151331.

https://doi.org/10.1172/JCI151331. system, built by Second Sight Inc. As expected, electrical stimulation of individual electrodes elicited phosphenes with locations that corresponded to the retinotopic map in the visual cortex $(7,8)$. However, when multiple electrodes were stimulated at the same time, the perceptions typically merged into larger phosphenes, making shape recognition practically impossible. This could be related to the fact that surface electrodes interact with relatively large volumes of cortex, resulting in a low spatial resolution of the perceived phosphenes. Moreover, the currents reported for evoking perception were on the order of several milliamperes (7). Such large currents could cause kindling of the cortex and eventual seizures, especially when groups of electrodes need to be stimulated simultaneously to create useful phosphene percepts. To circumvent these limitations, Beauchamp et al. developed two innovations: current-steering procedures and rapid sequential stimulation of electrodes to produce a sequence of phosphenes that traces out the shape of the intended pattern. However, although current steering and sequential stimulation can help to improve the utility 
of a cortical visual prosthesis with surface electrodes, there are still a number of challenges to overcome $(8,9)$. For example, every pulse train on a given electrode had to be completed before a pulse train on a second electrode could begin. Hence, dynamic stimulation was limited to only one phosphene at a time, and it remains unclear whether multiple phosphenes can be presented at the same time. Another limitation is the difficulty of conveying information about visual objects that move or change shape, because drawing a single shape takes time.

The work of Schmidt et al. (10) showed that microstimulation via microelectrodes that penetrated the visual cortex could help to mitigate some of these limitations. Phosphenes could be evoked with stimulation currents in the tens of microamps range, and stimulation via electrodes that were spaced close to each other evoked separate phosphenes. However, in the study by Schmidt et al., electrodes were implanted individually and many broke early in the experiment; therefore, only very limited testing of pattern recognition could be conducted.

We propose that arrays of penetrating electrodes such as the Utah Electrode Array (UEA) might form the foundation for the restoration of a useful visual sense (11-13). Recent results in monkeys support this point of view and show that the conjoint stimulation of multiple UEAs implanted in area V1 allowed the recognition of simple shapes, motions, and letters (14). However, a number of questions remain regarding the use of the UEA for a cortical visual prosthesis. For example, can a UEA be implanted and explanted in the visual cortex of a blind human subject without complications? Is the stability of the implanted UEA interface sufficient to support long-term use? What fraction of the implanted electrodes will yield visual percepts in a blind human? What stimulation levels are required to evoke visual percepts? Are phosphene-inducing stimulation currents within safe levels, avoiding damage to the cortical tissue and the UEA? Can the microelectrodes be implanted in extrastriate areas? And finally, do 2D spatial and temporal stimulation patterns evoke discriminable patterned percepts or just large, amorphous blobs of light?

Here, we report experiments conducted over a 6-month period in a long-term blind human subject, who had no light perception over the last 16 years. Single-unit recordings were possible, and stimulation thresholds that evoked phosphenes were within safe levels and remained stable over the whole experimental period. Simple forms of spatially patterned electrode stimulation evoked discriminable patterned percepts, allowing the blind participant to identify several letters and recognize object boundaries. Furthermore, we observed a learning process that helped the subject to recognize stimulus patterns across repeated presentations. These are encouraging short-term results in a single subject. However, further investigations in more subjects and over a longer period of time are required to determine whether a limited but useful sense of vision to the blind can be provided by intracortical microelectrodes.

\section{Results}

Electrode targeting and surgical implantation. The study protocol was approved by the Hospital General Universitario de Elche Clinical Research Committee (see Methods). A UEA (Blackrock Microsystems) was used, and its 1.5-mm-long electrode shanks were arranged in a $10 \times 10$ grid pattern $(11,15)$. The numbering scheme that identifies individual electrodes is shown in Figure 1A. Electrode tips were coated with sputtered iridium oxide to improve their charge injection capacity (16). The UEA was implanted in the subject's right occipital cortex, near the occipital pole and close to the border between V1 and V2 (see Methods and Figure 1, B and C).

Implant stability. Following UEA implantation, the mean electrode impedance increased (particularly during the first 15 days) and then decreased back to baseline values (Figure 1D). For each experimental session, there were only minor variations in the impedances of all 96 electrodes, with most of the impedances being in the range of 37 to $56 \mathrm{k} \Omega(47 \pm 4.8 \mathrm{k} \Omega$; mean $\pm \mathrm{SD})$.

Each experimental session was preceded and concluded by a 2-minute period of spontaneous neural recordings. The neural activity appeared to be reliable and repeatable on a day-to-day basis. The number of electrodes that yielded multiunit activity varied from day to day, but some electrodes recorded reliable spikes approximately $65 \%$ of the time over the 175 days of the study. Figure $1 \mathrm{E}$ shows 3 representative sets of recordings obtained on days 3,77 , and 154 , and the summary recording statistics for each electrode over the 175 experimental days. The colors in this figure represent the number of days on which more than 50 reliable spikes were recorded on a given electrode over the 2-minute recording period (out of 50 recording sessions). Most viable electrodes had signal amplitudes of $60 \mu \mathrm{V}$ and noise amplitudes of less than 50 $\mu \mathrm{V}$. Signal-to-noise ratios (SNRs) greater than 2 were required for the spikes to be included in the analysis and spike amplitudes over $900 \mu \mathrm{V}$ were frequently observed. The SNR of the recordings did not change significantly over time $(P=0.294)$.

Spontaneous and electrically induced phosphenes. The subject experienced spontaneous positive visual phenomena (phosphenes) prior to implantation, as is common in many blind persons (17-19), although these are often under reported or under recognized. After UEA implantation, the subject experienced a transient increase in spontaneous phosphenes that could appear at any time during the day and that did not correlate with an increase in the multiunit activity recorded by the implanted microelectrodes. In some experiments, we performed simultaneous EEG recordings and we did not observe any epileptiform or pathological activity associated with the perception of spontaneous phosphenes.

The number of these spontaneous phosphenes decreased gradually over time. In the first days after implantation, the average number of phosphenes was approximately 1 every 5 to $10 \mathrm{sec}-$ onds. Six weeks after the implantation, the frequency was reduced to approximately 1 phosphene every 30 to 60 seconds, and after 12 weeks she reported episodes of spontaneous phosphenes only very occasionally.

Before electrically induced visual perceptions could be studied, we had to ensure that the subject was able to reliably discriminate electrically induced phosphenes from the spontaneous phosphenes described above. Since the features of both spontaneous and electrically induced phosphenes were very similar, early in the experimental period it was very difficult for the subject to discriminate them. After training, the subject began to correctly discriminate electrically induced from spontaneous phosphenes. Two key issues facilitated her ability to discriminate phosphenes. First, 

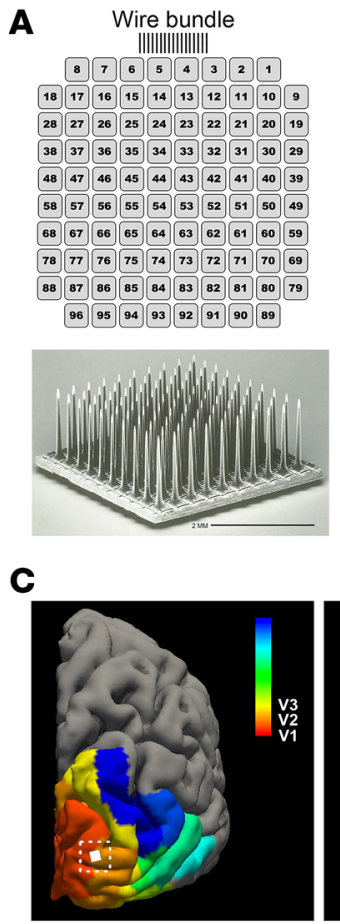

Visual areas

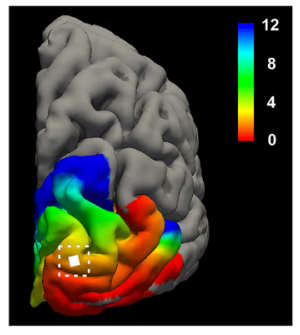

Eccentricity

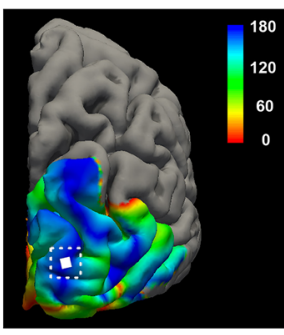

Polar angle

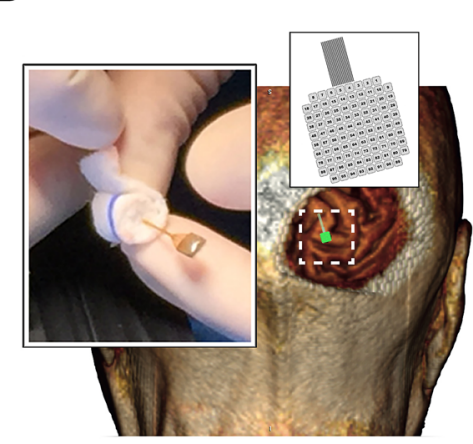

D

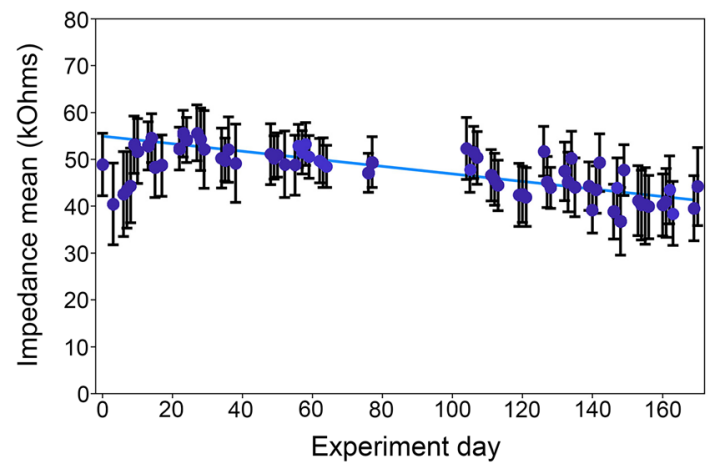

E

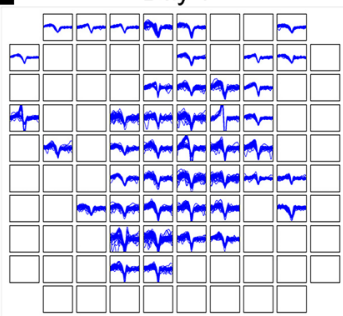

Day 154

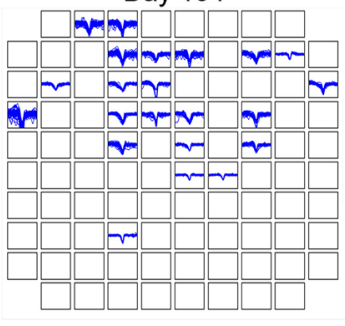

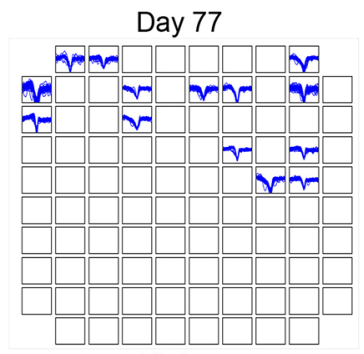

All days

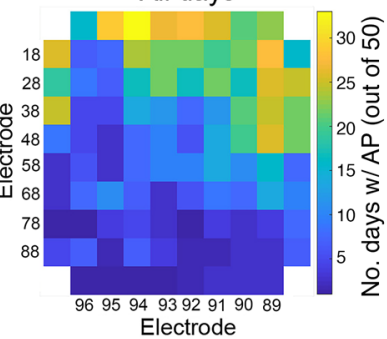

Figure 1. Utah Electrode Array (UEA) implantation and electrophysiological recordings. (A) Scanning electron microscopy image of the UEA and numbering system used to identify specific electrodes (electrode side shown). (B) Location of the UEA implantation site on the right occipital cortex. Inset: Image of the UEA to be implanted during surgery. (C) Predicted retinotopic map organization superimposed on the 3D reconstruction of the volunteer's cerebral cortex with the implantation site indicated (left, location of visual areas; middle, eccentricity; and right, polar angle). (D) Average electrode impedances across the 6 -month study period. The mean impedances increased by $20 \%$ in the first week, and gradually decreased toward their initial values (blue line). (E) Examples of recorded waveforms on days 3, 77, and 154 with summary statistics of recorded multiunit responses. Color in the heatmap represents the number of days on which more than 50 reliable action potentials were recorded on a given electrode over the 2-minute recording period.

during the training phase, the subject began to understand that all evoked phosphenes were small spots of light, and that they always appeared in conjunction with a low-frequency "stimulating-now" tone that was used to indicate the start of electrical stimulation. Further, she learned that the evoked phosphenes were always localized to the same general region of her visual space; thus, the subject could recognize them more easily.

To verify that the subject was correctly discriminating stimulus-evoked and spontaneous phosphenes, a sequence of superthreshold stimuli interspersed randomly with sham trials was delivered to the subject, with a "stimulating-now" tone indicating the delivery of each stimulus (real or sham). The subject indicated phosphene perception with a button press. Over the course of 4 days, 1000 superthreshold stimuli interspersed with 500 sham trials were delivered to different microelectrodes. The subject correctly identified 947 of the superthreshold stimuli, and only infrequently (23 out of 500) indicated perception of an evoked phosphene when a sham trial was presented. We also used a 2-alternative forced-choice (2-AFC) paradigm where the subject had to indicate if the first or the second stimulus was associated with a phosphene. The percentage of correct responses in these experiments was greater than $95 \%$. Her reliable performance in these experiments allowed us to begin the optimization of the various electrical stimulation parameters.

Stimulation parameter optimization. Since threshold currents for the detection of phosphenes are a function of multiple parameters (amplitude of the biphasic stimulus, pulse frequency, pulse duration, inter-phase interval, train length, and polarity of the stimulus), we initially performed several experiments to optimize these parameters. Optimum stimulation was defined as the combination of stimulation parameters that provided reliable perceptions with the minimum amount of current in a given stimulation period. Current-induced tissue damage during electrical stimulation was avoided by keeping stimulus charge density below the damage threshold boundary $(20,21)$. In order to reduce response variability, we performed these optimization experiments by 
A

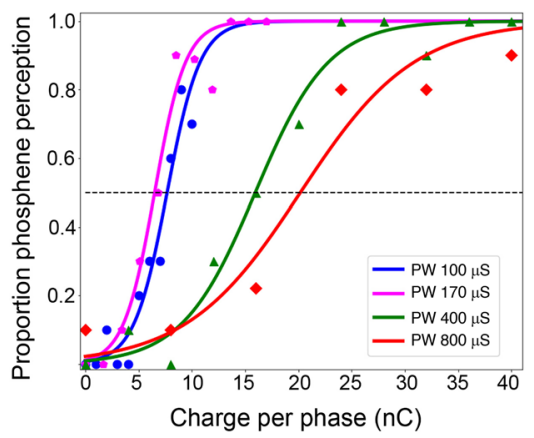

B

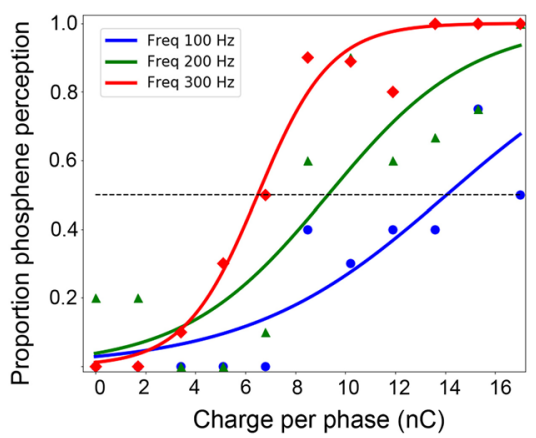

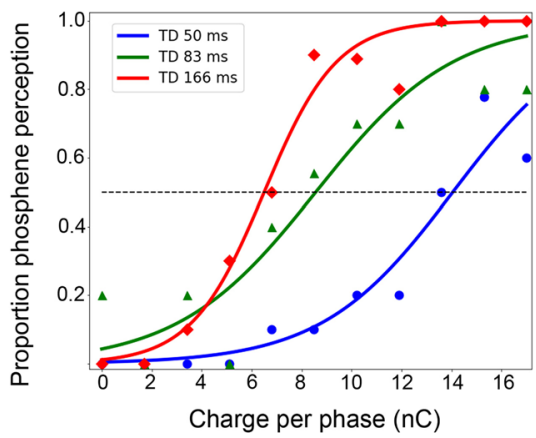

Figure 2. Relationship between phosphene thresholds and charge per phase. (A) Probability of phosphene perception for different pulse durations (pulse width, PW). (B) Effects of the stimulation frequency. (C) Effects of the duration of the train (TD) of stimulation pulses. One hundred twenty responses were used for each psychometric curve. The dashed horizontal blue lines represent the thresholds (50\% probability of detection).

simultaneously passing the same currents via 4 neighboring (abutting) electrodes.

Representative psychometric curves of these optimization experiments are shown in Figure 2, which shows the probability of phosphene perception as a function of the charge per phase for different pulse durations (Figure 2A), frequencies (Figure $2 \mathrm{~B}$ ), and train durations (Figure 2C). The lower charge per phase was reached using biphasic stimuli with phase durations of 170 $\mu \mathrm{s} / \mathrm{phase}$, although the behavioral results were similar for phase durations of 100 and $170 \mu \mathrm{s}(P=0.26$, ANOVA test). Increasing the phase duration to $400 \mu$ s or $800 \mu$ s resulted in a significant increase in the charge per phase. A relatively small amount of charge per phase was required when we used a frequency of 300 $\mathrm{Hz}$ and a train duration of $166 \mathrm{~ms}$ (Figure 2, B and C). The polarity of the stimulation (negative/positive phase first) did not have a significant influence on stimulation thresholds ( $P=0.108, t$ test).

Based on these results, we selected the following parameters for inducing phosphene percepts: stimulus trains composed of 50 charge-balanced cathodic first biphasic stimuli, a phase duration of $170 \mu \mathrm{s} /$ phase, an interphase interval of $60 \mu \mathrm{s}$, and a frequency of $300 \mathrm{~Hz}$ (train duration of $166 \mathrm{~ms}$; see Figure 3A). We used these stimulus parameters in most experiments (unless otherwise specified).

Stimulation thresholds. To measure phosphene thresholds, we used a binary search procedure (see Methods). We were able to reliably evoke phosphenes with 88 of the 96 electrodes. Mean stimulation thresholds for evoking phosphenes using single electrodes was $66.8 \pm 36.5 \mu \mathrm{A}$ (range $7-128 \mu \mathrm{A}$ ). Thresholds measured on different single electrodes ranged from $1.2 \mathrm{nC} /$ phase up to 20.4 $\mathrm{nC} /$ phase (average threshold of $11.2 \mathrm{nC} /$ phase), and threshold charge densities to evoke a visual perception at single electrodes (calculated from the geometric surface areas of the electrodes) ranged from 40 to $680 \mu \mathrm{C} / \mathrm{cm}^{2} /$ phase (average threshold charge density was $374 \mu \mathrm{C} / \mathrm{cm}^{2} /$ phase). Nonetheless, since we used sputtered iridium oxide film electrodes, in which the effective surface area is much larger than the nominal geometric surface area, it is highly probable that the charge densities in the tissue were lower.

Figure 3B shows the distribution of average thresholds and Figure $3 \mathrm{C}$ shows a representative histogram of the thresholds for all the individual electrodes in week 21, whereas Figure 3, D and $\mathrm{E}$ show the distribution of phosphene thresholds for groups of 4 electrodes distributed across the UEA. The threshold levels appeared to be distributed quite uniformly across the entire UEA. A longitudinal study of mean current threshold levels from the first reliable measurements showed that mean thresholds of individual electrodes increased by $19.4 \%$ over the last 4 months of the study, from $64.8 \pm 25.7 .5 \mu \mathrm{A}$ up to $80.4 \pm 28.8 \mu \mathrm{A}$ (Figure $3 \mathrm{~F}$ ). Therefore, thresholds were relatively stable over time (changes were less than $20 \%$ ), akin to the electrode impedances and the quality of multiunit recordings.

We also investigated phosphene thresholds by stimulating subsets of 2, 4, 9, and 16 contiguous electrodes at various locations across the UEA. We found only subtle differences between phosphene thresholds evoked by single electrodes and pairs of electrodes, but stimulation of larger groups of electrodes was associated with a significant reduction in thresholds $(P<0.001$, ANOVA test). Figure $3 \mathrm{G}$ shows 2 representative psychometric curves measured with single electrodes and 4 abutting electrodes. The mean thresholds for 4 -electrode stimulation were $28 \%$ lower on average $(48.0 \pm 22.6 \mu \mathrm{A})$ than those of single electrodes $(66.8 \pm 36.5 \mu \mathrm{A})$ and the threshold stimulation levels for the combinations of 4 electrodes were very stable over time (Figure 3F). Supplemental Figure 1 shows the mean thresholds for single electrodes and for groups of 2, 4, 9, and 16 contiguous electrodes.

Simultaneous stimulation and recording experiments. In some experiments, we performed electrophysiological recordings during electrical stimulation (see Supplemental Video 1). As expected, electrical stimulation often altered the activity of the neurons surrounding the electrodes. Figure 4 shows some representative examples. In general, the electrical stimulation trains were followed by an increase in multiunit activity (85\% of the cases). However, the increased firing of the neurons around the stimulated electrodes did not always correlate with the perception of phosphenes (Figure 4A). For trials in which the subject reported seeing a phosphene, we observed an increase in activity in $65.5 \%$ of the trials. The remaining $34.5 \%$ of trials showed an increase in activity trials without phosphene perception. On some occasions (15\% of trials), we also found an inhibition of spiking activity on some electrodes following electrical stimulation, which usually returned to baseline after a few seconds (Figure 4B). 
A

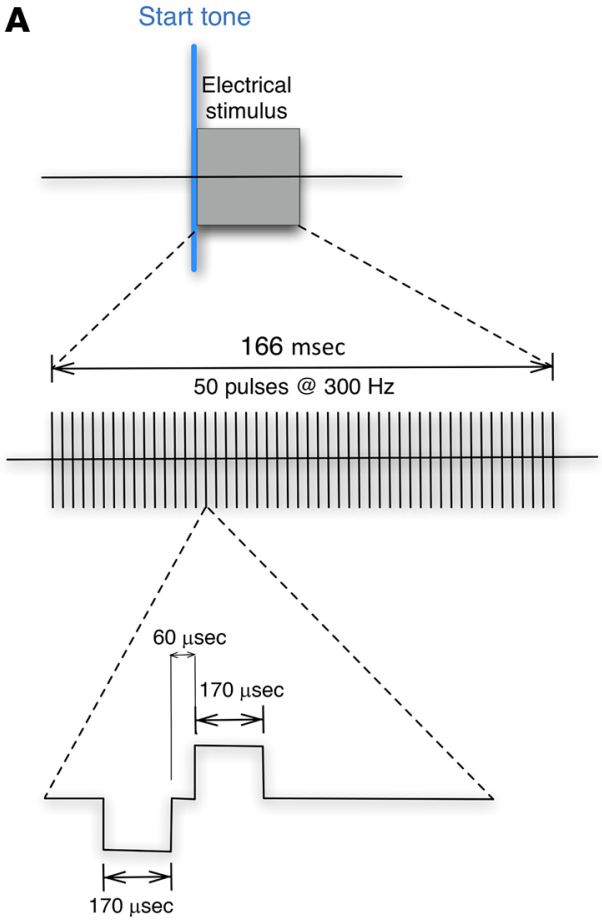

B

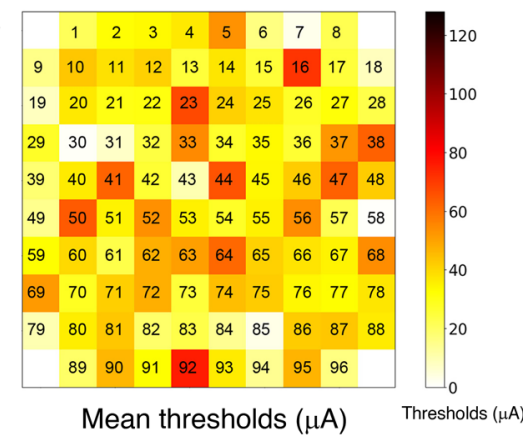

D

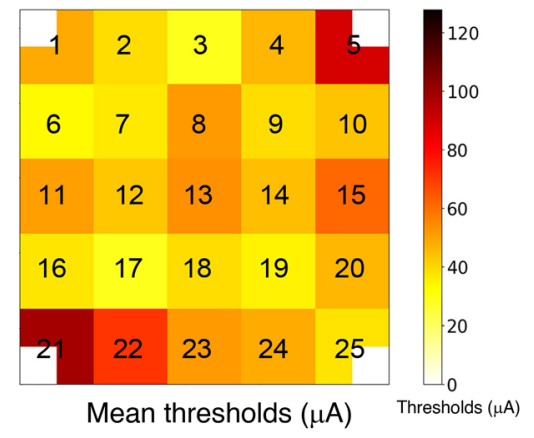

C

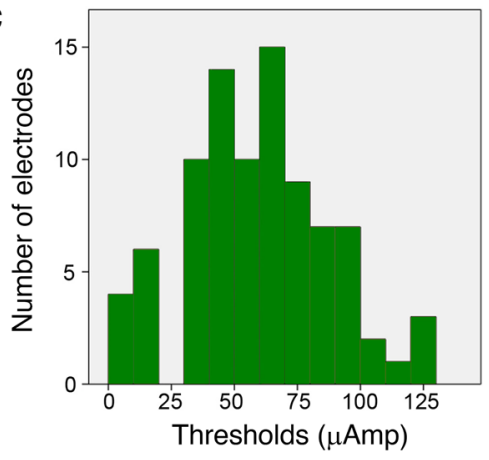

E

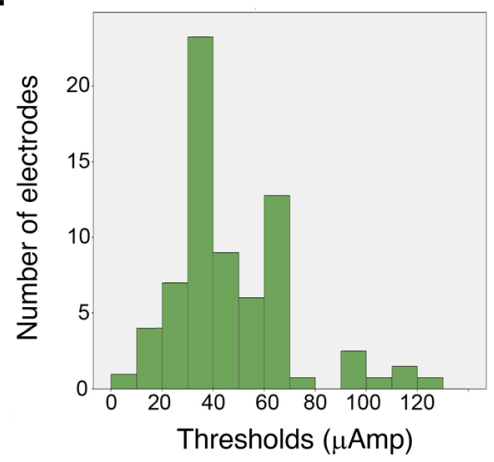

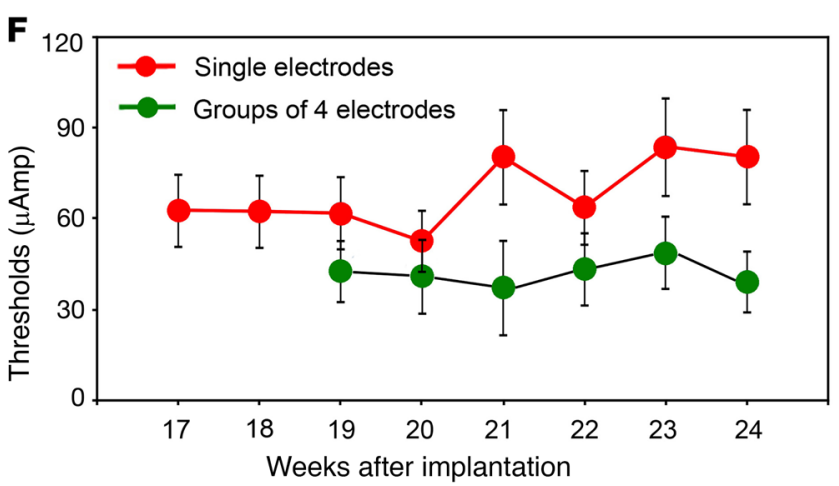

G

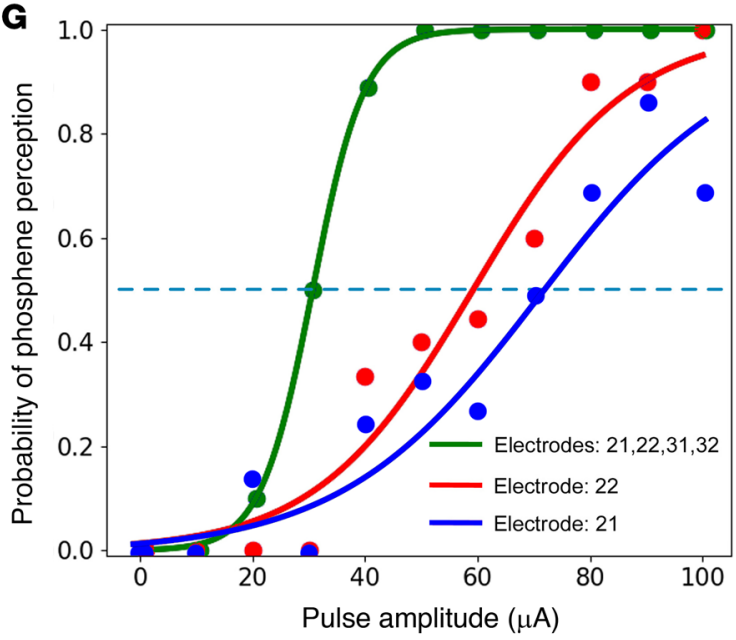

Figure 3. Phosphene thresholds. (A) Diagram illustrating the stimulation parameters of the biphasic pulse waveforms that were used for the quantification of the thresholds. (B) Distribution of phosphene thresholds for stimulation via single microelectrodes. Electrode tips are pointing away from the page. (C) Distribution of the thresholds for all the electrodes on week 21. (D) Distribution of phosphene thresholds evoked by simultaneous stimulation via sets of 4 contiguous (abutting) electrodes. Electrode tips are pointing away from the page. (E) Histogram of averaged thresholds for stimulation via the sets of 4 electrodes. (F) Evolution of the threshold for stimulation of single electrodes and for groups of 4 electrodes. Data presented as mean \pm SEM. (G) Representative psychometric curves generated in response to stimulation (biphasic pulses, with a phase duration of $170 \mu \mathrm{s} / \mathrm{phase}$ and a frequency of 300 $\mathrm{Hz}$ ) via 1 and 4 abutting electrodes.

When we stimulated several electrodes simultaneously, we observed diverse responses in different electrodes. Often there was an increase in activity in all the electrodes ( $84 \%$ of the trials), but sometimes we also recorded an increase in activity related to the stimulation in some electrodes and some inhibitory responses in other electrodes. In several cases (16\% of trials), we also observed inhibitory responses in all of the targeted electrodes, despite the fact that the subject reported seeing a weak percept.

Overall, $74.7 \%$ of stimulation trials that evoked an increase in the activity recorded around the electrodes (either by stimulating a single electrode or several electrodes simultaneously) were associated with a reported visual perception.

Phosphene mapping. During the 6-month study period we performed a total of 18 mapping sessions to estimate the location of the phosphenes in visual space. The subject manually indicated the location of the phosphene on a tablet with a small post in the center, which was used as the reference location (see Methods). An example map of the phosphene location of each individual microelectrode in the subject's visual field is shown in Figure 5A. All the phosphenes were close to the central visual field and 
A

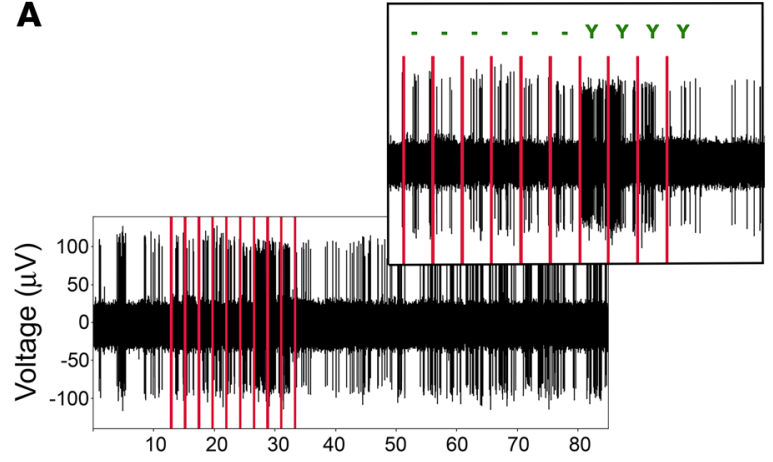

B

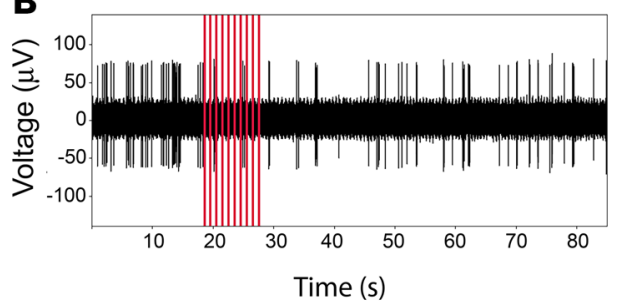

Figure 4. Representative simultaneous stimulation and recording experiments. (A) Repetitive stimulation of electrode 28 with $78 \mu \mathrm{A}$ at 0.5 $\mathrm{Hz}$ (red lines, 10 times, identical parameters) induced an increased firing of the neurons surrounding this electrode. Only the last 4 stimulations were associated with the perception of a phosphene (see upper enlarged panel where "-" indicates no perception and " $Y$ " indicates perception). (B) Repetitive stimulation of electrode 9 with $64 \mu \mathrm{A}$ at $1 \mathrm{~Hz}$ induced an inhibition of the neurons surrounding the electrode that recovered after a few seconds. In each of these examples, the neural recording was obtained from the stimulated electrode. Red vertical bars indicate the stimulus times.

located in an ellipsoidal region in her left hemifield (shown as a red ellipse in Figure 5A) adjacent to the horizontal meridian. However, the reported location of each phosphene varied slightly between sessions, especially for some of the electrodes (Figure $5 \mathrm{~B})$. These variations were always less than 0.5 degrees and were influenced by gaze position. There was also a good agreement between predicted phosphene locations based on our presurgical analysis (refs. 22, 23; yellow area in Figure 5A) and the reported locations of the evoked phosphenes (blue dots with electrode numbers in Figure 5A).

Phosphene color. When individual microelectrodes were stimulated at or above the subject's $50 \%$ threshold level (the stimulus that evoked a phosphene $50 \%$ of the time), the subject usually reported that the evoked perceptions were colorless (white). For current intensities below the $50 \%$ threshold, phosphenes were usually colorless, but sometimes the reported color was yellowish or sepia toned. When we used very low intensity currents $(<5$ $\mu \mathrm{A})$, the subject often perceived something resembling a very dim light, which she described as a "change of intensity." She was able to clearly differentiate these low-intensity perceptions from spontaneous and normally evoked phosphenes, but it was difficult for her to localize the exact position and size of these blurry perceptions in her visual field. Nonetheless, on many occasions she said that they were located "at the center of her left eye."

Modulation of phosphene brightness and size. We asked the subject to subjectively calibrate the brightness and size of her pho- sphenes on a scale from 0 to 5 . Phosphene brightness increased with higher currents $(P<0.001$, ANOVA test). Figure $6 \mathrm{~A}$ shows the average brightness ratings for 10 individual electrodes. The highest brightness ratings were reached with currents of approximately $90 \mu \mathrm{A}$, close to the largest currents that were tested. In another set of experiments, we investigated how the phosphene brightness depended on the phase duration of the biphasic pulses. We used 3 different phase durations: 100, 400, and $800 \mu$ s. While the perceived brightness increased slightly by increasing the duration of each phase of the stimulus, the changes were not significant $(P=0.727$, ANOVA test).

The estimated phosphene size evoked by single electrode stimulation was usually very small $(0.8 \pm 0.8$; mean \pm SD of subject's size estimates) and resembled "pin points" of light at arm's length, although there was considerable variability in perceived phosphene size evoked by different electrodes (size estimates ranged from 0.5 to 3.5 ; subjective units). The size of the evoked perceptions did not change significantly as a function of current amplitude on a given electrode $(P=0.212$, ANOVA test).

Increasing the number of stimulating electrodes significantly increased the subjective brightness and perceived size of the phosphenes (Figure 6, B and C). This relationship was observed when stimulation of groups of electrodes $(2,4,9$, and 16$)$ was compared to that of single electrodes $(P<0.001)$, and when stimulation of larger groups of 4,9 , and 16 electrodes was compared to that of smaller groups of 2 electrodes ( $P<0.001$, ANOVA test).

We also investigated the possible accommodation of phosphenes to repeated suprathreshold stimulation (see Methods). Our results showed that the apparent brightness and size of the phosphenes did not change significantly with 30 successive stimulations $(P=0.727$, Pearson's correlation coefficient $=0.010)$.

Two-point discrimination. A number of stimulation experiments were conducted with 50 pairs of electrodes (10 stimulations/trial). In $23.6 \%$ of the stimulations, the simultaneous stimulation of 2 electrodes evoked 2 discrete phosphenes, especially for widely separated electrode pairs. This occasionally also happened for electrodes separated by $400 \mu \mathrm{m}$ (Figure 7A). However, very often ( $76.4 \%$ of the stimulations), simultaneous stimulation of electrode pairs evoked a single phosphene, albeit with a subjective estimated size that was $1.9 \pm 0.1$ times greater than the estimated size of the phosphenes evoked by the stimulation of either electrode in isolation $(0.8 \pm 0.1 ; P<$ 0.001, ANOVA test). The results were generally consistent for the same pair and testing session.

To further quantify the subjective perception of phosphene size when stimulating pairs of electrodes, we examined 4 combinations of pairs of electrodes that had interelectrode distances of $400,800,1200,1600,2000,2400,2800,3200$, and $3600 \mu \mathrm{m}$. These experiments were performed with 36 pairs of electrodes (10 stimulations/trial). Our results showed that the subjective estimated size correlated well with the degree of separation between electrodes (Figure 7B). Furthermore, the subject reported that the percepts had more elongated shapes when we increased the distance between the stimulating electrodes. This suggests that the phosphene's size and appearance is not only a function of the number of electrodes being stimulated, but also of their spatial distribution in the UEA. 
A

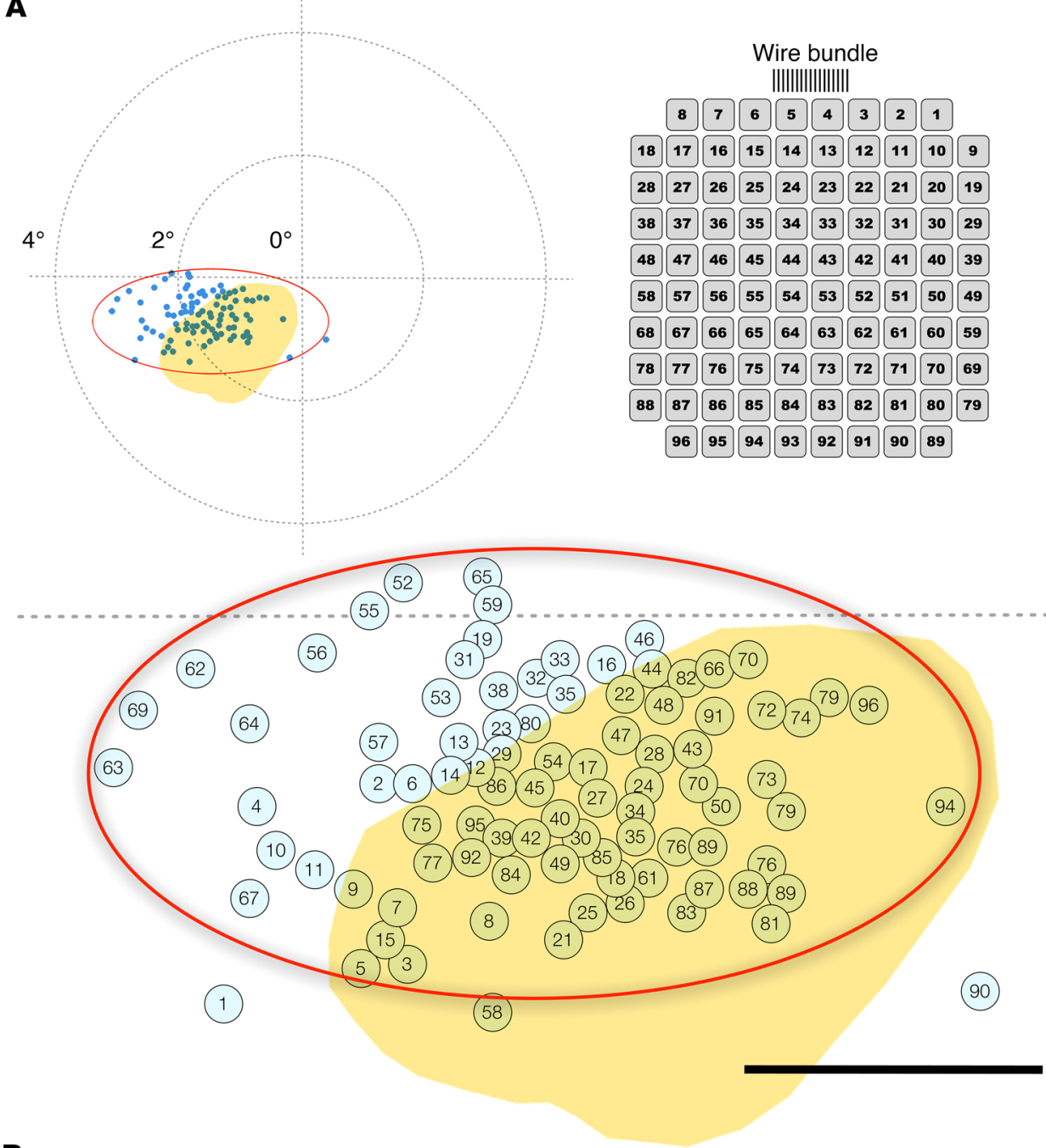

$0^{\circ}$

B

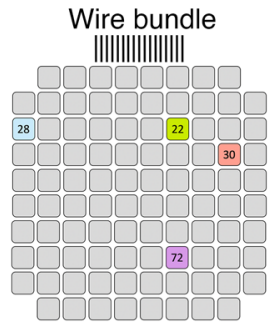

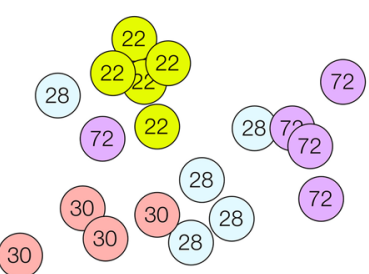

(30)

Figure 5. Phosphene map and relative brightness and size of perceived phosphenes. (A) Location of the perceived phosphenes (blue dots) and electrode numbering view from the pad side. The cross indicates the center of the subject's visual field (the intersection of her horizontal and vertical meridians). Yellow region: Expected location of the phosphenes based on a standard retinotopic map superimposed on the anatomy of the visual cortex of the patient using the procedures described by Benson et al. $(22,23)$ and the selected implantation site. Calibration bar $=1$ degree. (B) Changes in the location of the perceived phosphenes for the same 4 electrodes in 5 different trials. Calibration bar $=1$ degree.

We also stimulated 40 electrode pairs with a delay between stimuli to identify the minimum time interval that facilitates the perception of discrete phosphenes (10 stimulations/trial). For these experiments, we used a fixed pulse amplitude of $90 \mu \mathrm{A}$ for both electrodes and delays of 50,100, 200, 250,300, 400, 500,
$700,1000,2000$, and 4000 ms between the 2 trains of stimulating pulses. For electrodes separated by $400 \mu \mathrm{m}$, when stimulation of the 2 electrodes was temporally separated by more than $250 \mathrm{~ms}$, the subject perceived 2 different, distinct phosphenes $90 \%$ of the time. This discrimination reached $100 \%$ for electrodes separated 

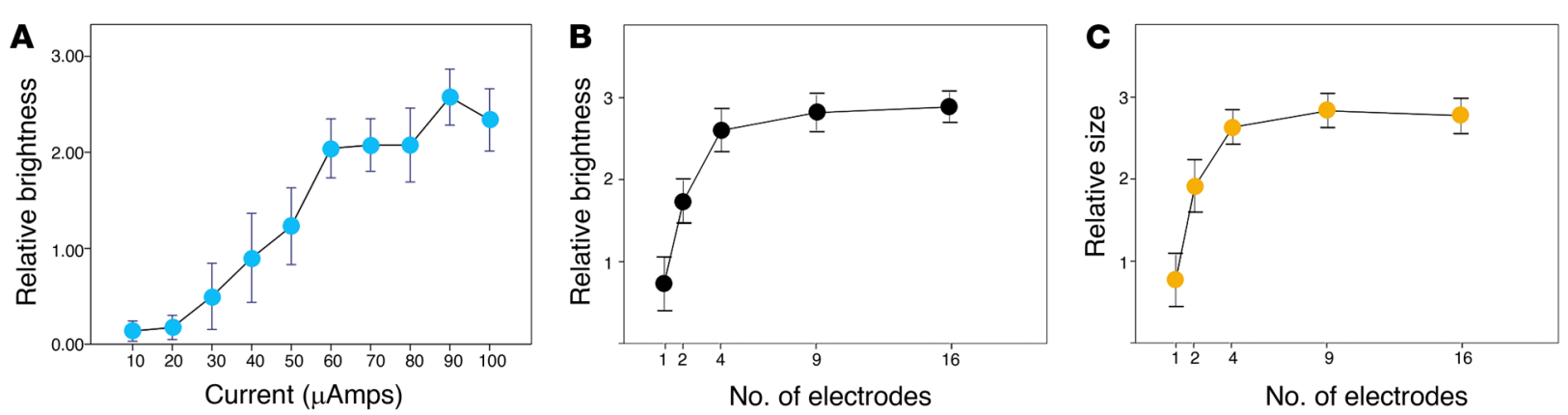

Figure 6. Relative brightness and size of perceived phosphenes on a subjective scale ranging from $\mathbf{0}$ to $\mathbf{5}$. (A) Averaged values of perceived brightness across 10 single electrodes. Maximum was reached for currents of approximately $90 \mu \mathrm{A}$. (B) Subjective brightness and (C) subjective size of perceived phosphenes as a function of the number of simultaneously stimulated electrodes. Error bars denote SEM.

by $565 \mu \mathrm{m}$ (along the diagonal) or further away. Further increasing the delay between both stimulus trains (from 300 to $4000 \mathrm{~ms}$ ) facilitated the identification of 2 distinct phosphenes.

Discrimination and recognition of complex $2 D$ stimulus patterns. We next investigated if the simultaneous stimulation of more than 2 electrodes induced more complex perceptions. For these experiments, we simultaneously stimulated different combinations of 3 to 16 electrodes distributed over the UEA. Increases in the number of simultaneously stimulated electrodes increased the probability of complex visual percepts. Furthermore, the subject reported that the perceptions elicited by groups of simultaneously stimulated electrodes were easier to perceive and clearer than when we stimulated single electrodes.

First, we simultaneously stimulated 30 different groups of 4 contiguous electrodes distributed across the UEA, plus 4 groups of 3 electrodes (at the corners of the array). Figure 7, C-E illustrate several representative examples of patterned stimuli that were used, and the subject's sketches of the 2D percepts that the stimuli evoked (drawn on a digitizer tablet). Some stimulation patterns evoked percepts of closely spaced dots (Figure 7C), whereas other patterns evoked horizontal or almost horizontal lines (Figure 7D). It was more difficult to induce vertical lines, but Figure 7E shows a representative example of 2 groups of 8 electrodes that evoked horizontal and vertical line percepts. To examine the reproducibility of the evoked percepts, and to monitor the subject's ability to discriminate between horizontal and vertical evoked percepts, we presented these 2 patterns in random order and asked the subject to indicate the perceived line orientation using a 2-AFC approach. The subject's performance was always above chance level and across 22 sessions on 4 different days, the overall success rate was $81.4 \%$. Moreover, her ability to correctly discriminate horizontal and vertical patterns increased with training, and in later sessions accuracy reached $100 \%$.

Encouraged by these results, in the last month of the experimental period we investigated several spatial stimulation patterns that we expected to induce the perception of letters using up to 16 simultaneously stimulated electrodes (the maximum number of simultaneous channels possible with our neurostimulator). Our subject spontaneously reported the perception of some characters and reliably discriminated some letters such as i, L, C, V, and O. Over a series of 5 days, we performed 24 sessions using a 2-AFC approach (30 stimulations/trial) to differentiate between several complex 2D patterns. The characters tested were i versus $\mathrm{L}$, i versus $\mathrm{O}$, $\mathrm{L}$ versus $\mathrm{C}, \mathrm{C}$ versus $\mathrm{V}$, and $\mathrm{L}$ versus $\mathrm{V}$. The overall success rate was always greater than $70 \%$. Figure $7, \mathrm{~F}-\mathrm{H}$ illustrate some of the electrode combinations that elicited the perception of letters. Although several combinations of electrodes evoked perceptions that resembled letters (such as an inverted $\mathrm{U}$ or a rotated $\mathrm{T}$ ), we were unable to induce the perception of all the letters of the alphabet. Furthermore, many stimulation patterns were not recognized as letters by our subject (see Supplemental Figure 2); hence, we only tried them a few times before switching to other stimulation patterns that were able to induce letter percepts. Additionally, on some occasions the perceptions did not match with our expectations based on the stimulated pattern of electrodes. An example is shown in Figure 7G, where we simultaneously stimulated a group of 4 electrodes and another separate group of 3 electrodes, and the participant's perception resembled the letter L.

To increase motivation and facilitate the learning of different stimulus patterns, the subject played several games that in reality were 2 -AFC experiments (20 stimulations/trial). The games used patterned stimulation, and the main aim was to help the subject to discriminate between different patterns and/ or group of electrodes. One of the games was to guess if Maggie Simpson (a fictional character in the animated television series "The Simpsons") was shooting to the left or to the right. Each shooting direction was associated with the stimulation of a particular set of electrodes, and shooting sequences were randomized. After each shooting, the subject had to a press the appropriate button (left or right) on a wireless keypad to stop the bullets (see Supplemental Video 2).

To further investigate the influence of learning, we selected electrode sets that evoked 2 different alphanumeric characters, a lowercase $\mathrm{o}$ and an uppercase $\mathrm{O}$, which were easily recognized by the subject even on the very first trials (Figure 8A). When we asked the subject to characterize these percepts, she clearly and spontaneously described the perception as a "big O" or a "small o." Across 20 sessions over several days, involving a total of 400 trials, there was an improvement process that reached $90 \%$ to $95 \%$ after 70 trials and $100 \%$ accuracy in the latest sessions (Figure 8B). Furthermore, the effects of training were lasting. These improvements in the identification of evoked perceptions over time indicate that the subject learned to reliably recognize specific patterns. 
A

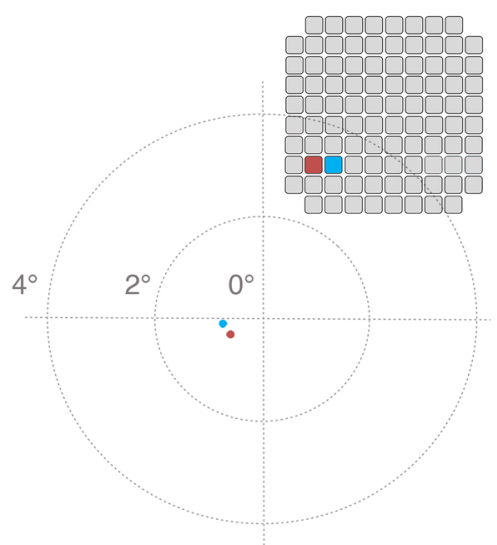

B

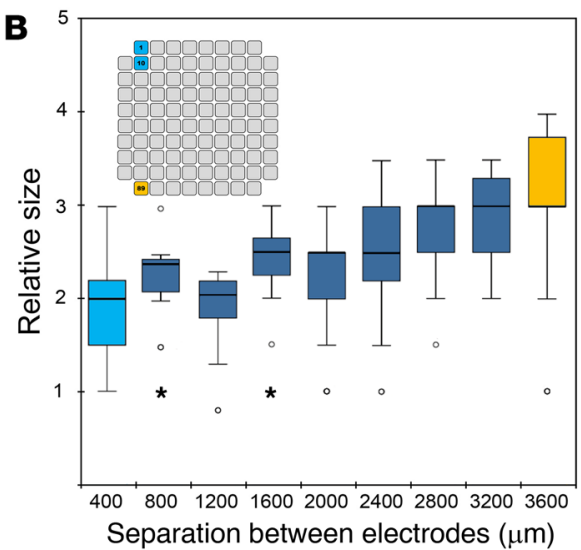

C

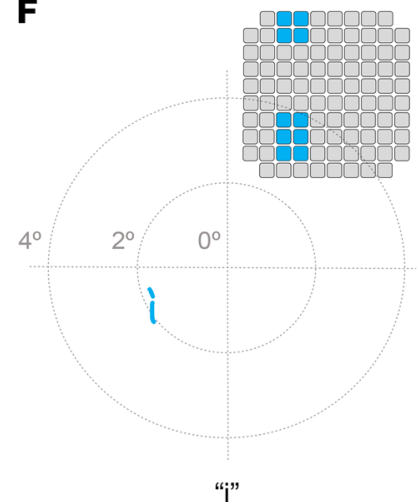

D

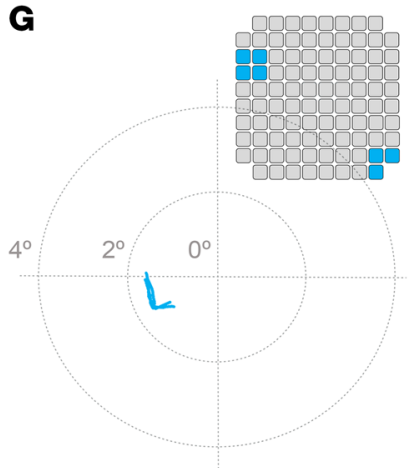

"L"

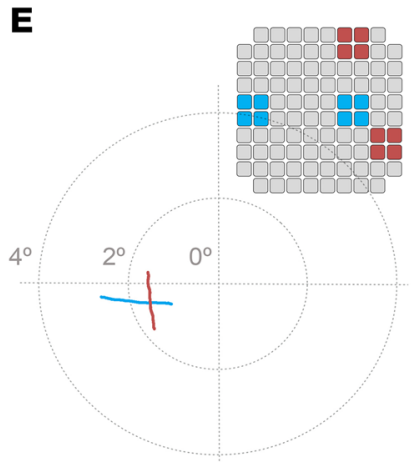

H

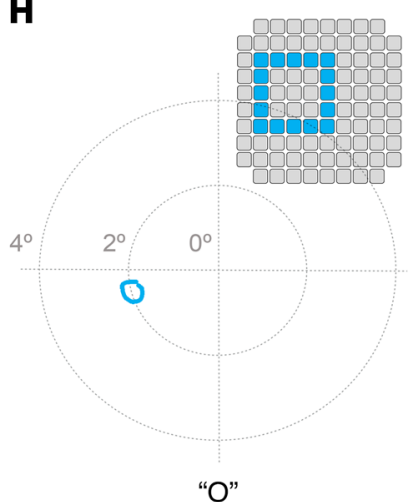

Figure 7. Examples of perceptions evoked by simultaneous stimulation of multiple electrodes. (A) The stimulation of the 2 electrodes shown in the inset electrode map induced the perception of 2 closely spaced phosphenes. (B) Box-and-whisker plot of subjective phosphene size for electrode pairs separated from 400 to $3600 \mu \mathrm{m}$. Inset: Relative location of electrodes 1 and 10 in the UEA, which are separated by $400 \mu \mathrm{m}$ (light blue color), and electrodes 1 and 89 , which are separated by $3600 \mu \mathrm{m}$ (orange color). In the box-and-whisker plot, the boundary of the box closest to zero indicates the first quartile, and the boundary of the box farthest from zero indicates the third quartile. The horizontal line is the median. The whiskers show the maximum and minimum values, with the exception of outliers (small circles) and extremes (stars). (C) Simultaneous stimulation of 4 contiguous electrodes was perceived as 3 small dots. (D) Stimulation of 12 electrodes induced the perception of a horizontal line. (E) Simultaneous stimulation of the 2 groups of 4 blue electrodes evoked the percept of a line with a horizontal orientation, whereas the simultaneous stimulation of the 2 groups of 4 red electrodes evoked the percept of a line with a vertical orientation. (F) Stimulation of these electrodes elicited the perception of a lowercase letter i. (G) Stimulation of these 2 groups of electrodes unexpectedly induced the perception of an uppercase letter $L$. (H) Stimulation of these electrodes elicited the perception of an uppercase letter 0.

Preliminary experiments with a bio-inspired retinal encoder. As the experimental period neared completion, we performed some preliminary tests using a bio-inspired retina-like visual encoder to dynamically stimulate different combinations of electrodes according to the visual scene in front of the subject. The visual encoder contained a head-mounted camera and spatial and temporal image processors (Figure 9, A-C). Using the visual encoder, the subject was able to "head scan" objects in front of her.
To investigate the spatial accuracy of this approach, the subject was first trained to use head scanning in order to discriminate the location of the borders between black and white bars printed on cardboard. The subject was able to locate the black/white border in all the tests (see Figure 9D and Supplemental Video 3). Then the subject was trained to discriminate the location of a large white square $(20 \times 20 \mathrm{~cm})$ appearing randomly at either the left or right half of a 21-inch computer monitor. The subject was seated $50 \mathrm{~cm}$ 
A
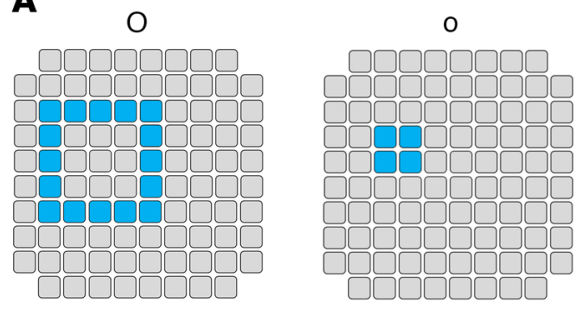

B

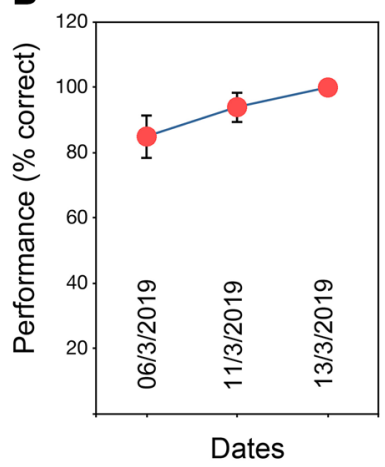

Figure 8. Evolution of the performance in a task in which the subject discriminated an uppercase $\mathbf{0}$ from a lowercase o. (A) Stimulated electrodes for the perception of each pattern. (B) Learning process that reached an accuracy of $100 \%$ in the last session. Error bars denote SEM.

directly in front of the monitor and quickly learned to perform head scanning to successfully localize the white square $(100 \%$ accuracy across 70 trials). We then presented a smaller white square $(14 \times 14 \mathrm{~cm})$ randomly in 1 of 4 possible locations - upper left and right and lower left and right - and she had to point out the location of the white square. The subject was able to correctly point to the white square $100 \%$ of the time (150 trials across 5 days), and her reaction time decreased from $8.6 \pm 0.6$ seconds for the first trials to $5.4 \pm 0.3$ seconds ( $38 \%$ less on average) after only 2 training days. The effects of training were long lasting and once she learned to perform the task, the required time remained stable (Figure 9E). Furthermore, after the short training period, the subject reported that the task became easier to carry out.

Explantation of the microelectrode array. After completing the 6-month study period, the implanted electrode array and the external connector were explanted without complications. The subject has been followed periodically after the explantation and reports no side effects or complications. The only observation reported by the subject was a new increase in the number of spontaneous phosphenes immediately after the explantation of the array, which lasted around 3 weeks.

\section{Discussion}

Individuals with profound blindness generally do not live in a world of total blackness but experience a variety of spontaneous phosphenes $(18,19)$ that should be considered in the development of any visual prosthesis. The initial inability of our subject to discriminate spontaneous from evoked phosphenes posed a major impediment to the performance of meaningful stimulation experiments. Other studies investigating cortically based sight restoration have reported similar observations $(10,24)$. Two months of almost daily experimentation were required before our subject was able to reliably discriminate evoked from spontaneous phosphenes. Any future human experimentation or any eventual clinical applications will have to take this initial learning period into account.

The results of our study, which was conducted over a 6-month period, show that implantation and explantation can be done without complications, implying that this procedure can be performed safely. Electrical stimulation evoked phosphenes on 88 of the 96 electrodes, and the mean stimulation threshold for evoking phosphenes was $66.8 \pm 36.5 \mu \mathrm{A}$, a value within the safe levels for neural stimulation. Furthermore, the demonstrated stability of the UEA as a neural recording and neural stimulating device was encouraging. Thus, we were able to record multiunit activity from most of the electrodes throughout the entire study period. In addition, the SNR and the noise level of the 2minute recordings performed every day did not change as a result of stimulation sessions and remained stable over time. This suggests that the implantation of the UEA and the stimulation over a period of 6 months neither impaired the function of neurons in close proximity to the electrodes nor affected the function of the underlying cortex.

In this work, we focused mainly on the optimization of electrical stimulation parameters and on longitudinal studies that investigated phosphene thresholds and their associated features (i.e., intensity, size, spatial location). The impedance of the electrodes and stimulation thresholds did not change by more than $20 \%$ over time. Although the duration of this study was limited to 6 months, UEAs have been successfully implanted in human subjects in numerous studies (25-29) and these devices have continued to work for many years. A recent systematic review of human neuroprosthetic research using the UEA reported that the mean number of days of UEA implantation across all participants at the time of the study was at least 578 days (30), and several studies indicate that UEAs retain their functionality in humans for more than 1000 days $(29,31)$. The results therefore suggest that silicon-based, penetrating microelectrode arrays could provide the stability required in a clinical device (11, 32-34) but further investigations in more subjects and over a longer period of time are needed.

A key issue with intracortical microstimulation of visual areas, which is only addressable in humans, is related to the potential complexity of the evoked percepts. Because the currents required for the production of phosphenes with intracortical microelectrodes are 2 to 3 orders of magnitude smaller than those required to evoke phosphenes using surface electrodes, microelectrodes can be placed closer together, providing better spatial resolution. Our results show that intracortical electrodes that were spaced as little as $400 \mu \mathrm{m}$ apart can generate separate perceptions. This 2-point resolution is closer than the $500 \mu \mathrm{m}$ resolution reported previously with a single electrode pair of gold wire electrodes (10), and about 5 times closer than has been achieved with surface electrodes $(2-5,7)$. Nonetheless, the perceptions evoked in this study via separate electrodes were often very close together, and simultaneous stimulation of 2 electrodes often evoked a single phosphene, albeit with a larger size. Schmidt et al. reported similar findings (10), which suggest that when multiple electrodes are simultaneously stimulated some specific characteristics of the individual phosphenes can be lost. In our study, although the phosphenes were in the expected global location based on our presurgical studies, they did not appear to follow a clear linear or 


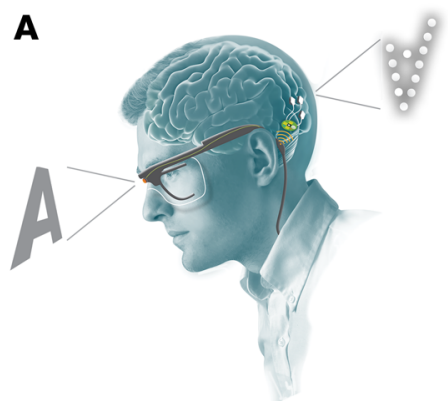

D

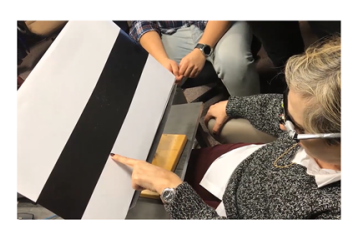

B

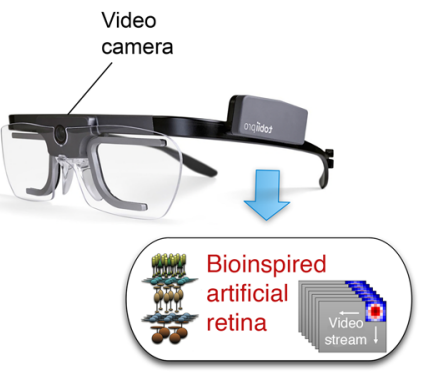

$\mathbf{E}$

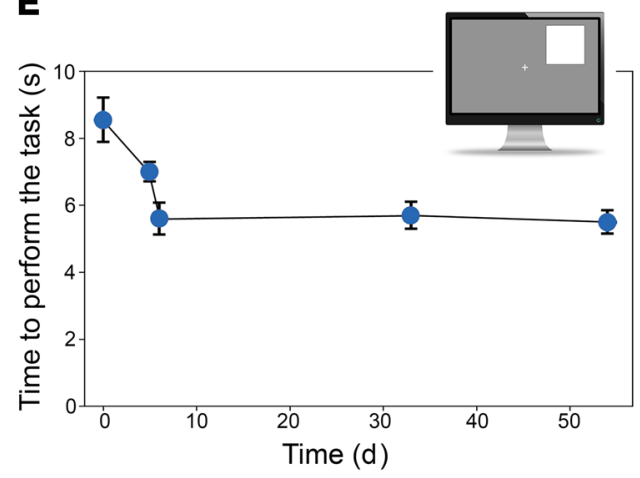

C
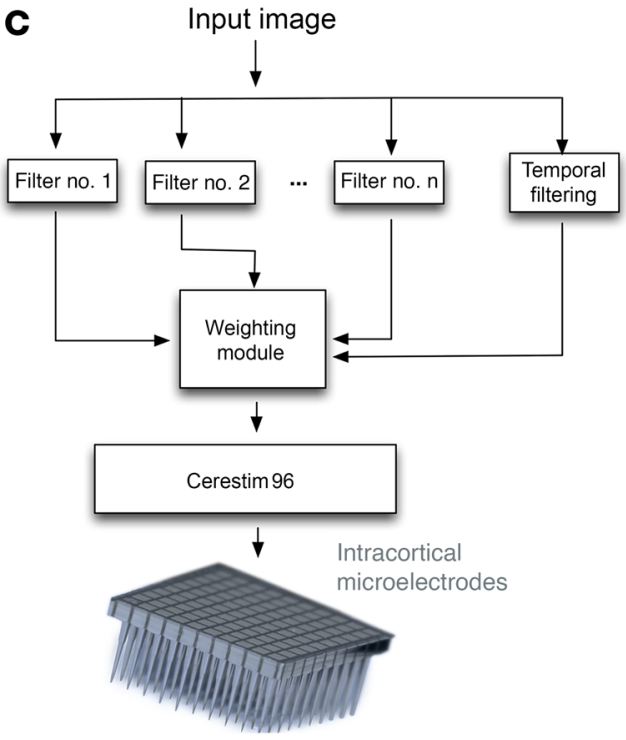

Figure 9. Bio-inspired artificial retina. (A) Schematic organization of the sight restoration concept. (B) The image acquisition system. The input images are captured by a video camera attached to a spectacle frame for subsequent bio-inspired processing. (C) Signal processing module. Input images are processed by a combination of several spatial and temporal filters that enhance specific features of captured information. The weighting module re-encodes this information into a neuromorphic stream of electrode addresses and sends it to the CereStim 96, which generates the stimulation signals applied to the intracortical microelectrodes. (D) Discrimination of the border between black and white bars using the bio-inspired artificial retina (frame extracted from Supplemental Video 1). (E) Evolution of the time required to perform the object location task (4 possible locations) over several days.

conformational map. We found similar results in previous animal experiments (35), and the visual prosthesis work with intracortical microelectrodes of Schmidt and colleagues also indicates that there is some variability phosphene location (10). This implies that although one can predict the average location of a phosphene, the actual locations exhibit some scatter, and a cortically based visual prosthesis may require spatial remapping or fine calibration.

Our blind volunteer was able to spontaneously discriminate several complex patterns and even to identify some letters with minimal training, which is advantageous for a visual prosthesis. However, when stimulation was delivered via multiple electrodes simultaneously, the evoked perceptions sometimes did not show a clear correlation with the relative locations of the stimulated electrodes. This may be due to the complex visuotopic organization of visual cortex, the implantation of a relatively small region of cortex with a single array $(3.6 \times 3.6 \mathrm{~mm})$, the spreading of conductive currents inside the cortex, or the stimulation of inhibitory cells and/or nearby axons. Furthermore, although the location of phosphenes was generally consistent with the retinotopic organization of visual cortex on a large scale, our results did not reveal a clear rectilinear transformation of the location of the evoked phosphenes and the position of each electrode. This could be due to either a fine-scale disorganization of the retinotopic map or to inaccuracies or biases in reporting phosphene locations. This implies that a functional cortical visual prosthesis may require some form of spatial remapping between visual input and electrical stimulation patterns. Further, when multiple electrodes are simultaneously stimulated, some of the distinctive characteristics of the individual phosphenes can be lost. Therefore, the perceptions induced by intracortical microstimulation appear to be more complex than initially believed and the generation of arbitrary patterns or recognizable letters is not a simple matter. Consequently, a key challenge for the future development of cortical visual implants is the delivery of interpretable information to the brain (9). In addition, most current coding strategies are only aimed at addressing spatial details, but we should also pay attention to other relevant visual attributes such as receptive field size, orientation, and movement. Moreover, we should also consider possible differences in coding among subjects as well as minimize (or eliminate) the need for recalibration.

Previous attempts to develop a cortical visual prosthesis have focused on implanting the electrodes in striate cortex (V1), which is involved in the initial processing of all visual information. However, many parts of V1 are difficult to reach because they are buried in the calcarine sulcus, which contains numerous blood vessels that supply the region and need to be avoided $(36,37)$. Therefore, extrastriate areas might also be suitable for implantation (37). Previous studies in monkeys and humans demonstrated that the thresholds for phosphene perception in areas V1 and V2 are similar and that the quality of the percepts that are elicited are comparable $(14,38-$ 40). Our present results support previous findings $(5,10,38,39,41$, 42) and show that the border between V1 and V2, which is located near the occipital pole, is a suitable place for a cortical visual prosthesis (37). However, a single UEA in this region is unlikely to be sufficient for useful vision; hence, in the future, we expect that several arrays of intracortical microelectrodes would be tiled across 
the visual cortex (43), permitting phosphene induction across a larger area of the visual field and forming the basis for functional sight restoration. For example, Cha et al. (44) and Dagnelie et al. (45) have shown that approximately 700 electrodes could provide a blind volunteer with useful visually guided mobility. Multiple tethered wired UEAs have been already implanted in the visual cortex of monkeys $(14,40,46)$ and these UEA implants allow monkeys to recognize simple shapes, motions, and letters (14).

The magnitude of currents required to excite cortical neurons and to evoke phosphene percepts could represent a safety concern in the development of a useful clinical device. Over the course of this study thousands of stimulus trains were delivered via the electrodes. We observed no evidence of neural kindling or the generation of epileptic seizures, and the participant did not report negative side effects resulting from the electrical stimulation. The work of Shannon (21), McCreery et al. $(47,48)$, and Cogan et al. $(20,49)$ established some guidelines for stimulation levels that are regarded as safe both for the stimulated tissues and the materials of the stimulating electrodes. The stimulation thresholds shown in Figure 2 are within these safety limits of stimulation. However, when multiple electrodes are stimulated in a simultaneous fashion, the currents will summate and can reach larger aggregate values. To mitigate this problem, more complex patterned stimulation using temporal interleaving of stimuli may be required to evoke $2 \mathrm{D}$ visual patterns. The exact stimulation strategy that could be used to achieve this is has yet to be resolved. Nevertheless, our subject reported seeing simple patterns and reliably discriminated some letters, and was able to correctly localize visual objects when multiple electrodes were stimulated simultaneously. These results highlight the potential of intracortical microstimulation to restore functional vision in the blind.

A clinical cortical visual prosthesis would be expected to use wireless actuation of the electrode arrays, and although wireless versions of the UEA and other systems have been developed (31, $50,51)$, these prototype systems are not yet sufficiently robust for clinical use. When such robust wireless systems become available, it is reasonable to expect that at least 7 to 10 wireless UEAs could be implanted in the time that it took to implant a single wired UEA. However, such a large number of implanted electrodes may pose data-transmission and stimulation problems in a wireless device, and these may be key factors limiting the number of UEA implants (34).

The results from this study suggest that even a relatively simple cortical prosthesis based on microstimulation via arrays of intracortical microelectrodes and a retinotopic encoding system can allow the recognition of some letters and the localization of shapes. Such a system could help to enhance safety in navigation and provide greater confidence for individuals with profound blindness in many environments. The findings reported herein also highlight the role of training to achieve desired behavioral outcomes. However, more experiments conducted over a longer period of time are still needed to achieve the clinical goals envisioned by these technologies.

\section{Methods}

Study design. The purpose of this study was to investigate the feasibility of a cortical visual prostheses for the blind based on intracorti- cal microelectrodes. Experiments were conducted on a 57-year-old, right-handed female, who had toxic optic neuropathy and complete blindness (no light perception) for the past 16 years. She had good cognition and functional abilities, was a skilled user of accessible technology for blindness, and a braille reader. Before her enrollment in the study, a complete medical and psychological evaluation was performed. This evaluation included a comprehensive medical history, a complete systemic, ophthalmological, neurological, and psychological evaluation, and a systematic mapping of the visual sensations induced by noninvasive stimulation of the subject's occipital cortex using transcranial magnetic stimulation (52).

Following the loss of her vision, the subject experienced episodic perceptions of elementary nondisruptive positive visual phenomena (PVP). She described these as flashing lights, blinking spots, or horizontal or vertical lines of various widths and lengths, some curved and some straight. She often perceived these visual phenomena as being localized to one or both eyes. They were more commonly associated with loud sounds and/or startle situations. It was very difficult for the subject to quantify the number and nature of these PVPs before her enrollment in the study, since over the years, she had learned to ignore their presence. Nonetheless, she was not distressed by these spontaneous phosphenes and always recognized these visual perceptions as unreal phenomena.

Implant location. The UEA was implanted in the subject's right occipital cortex, near the occipital pole and close to the border between V1 and V2 (Figure 1, B and C). During preliminary studies we found that transcranial magnetic stimulation of the right visual cortex elicited reproducible phosphenes in our subject, especially when the coil was located near the occipital pole. Therefore, we selected her right occipital cortex for implantation. For fine presurgical planning, we performed a 3D reconstruction of the surface anatomy and the neurovascular structures of the subject's primary visual cortex to optimize the implantation location of the electrode array. We tried to avoid as much as possible the major blood vessels that supplied the region. Anatomical images of the occipital cortex and other areas of the visual system were acquired on a 3-T magnetic resonance scanner (Siemens MAGNETON Skyra) using the MPRAGE protocol (192 sagittal slices, $256 \times 256$ matrix, $1 \times 1$ $\times 1 \mathrm{~mm}^{3}, \mathrm{TR}=1900, \mathrm{TE}=2.49, \mathrm{FS}=3$ ). We used Horos, a free and open-source computer software that is distributed under the LGPL license (https://horosproject.org/), to create 3D reconstructions of the cerebral surface showing cortical anatomy as well as the major cortical vessels. The Freesurfer image analysis suite v6.0 (https:// surfer.nmr.mgh.harvard.edu/) was used for cortical reconstruction and volumetric segmentation. Furthermore, we examined the predicted retinotopic organization of the occipital cortex of this blind subject using cortical surface anatomy to predict her visual areas $\mathrm{V} 1, \mathrm{~V} 2$, and V3 according to the procedures described by Benson et al. $(22,23)$. Based on this information, we selected a region at the border of V1 and V2, which could be accessed easily while avoiding major blood vessels.

We selected this region because the striate cortex is located in the medial surface of the occipital lobe, and hence more difficult to reach, and further, contains the calcarine artery, a branch of the posterior cerebral artery that could be damaged by the implantation. Moreover, our previous studies in people suffering from epilepsy and brain tumors who had to undergo surgical resection of a brain region show 
that electrical stimulation of extrastriate cortex (Brodmann area 18 and all Brodmann area 19) still induces visual perceptions with consistent retinotopic organization (37)

Surgery. The surgical procedures for UEA implantation followed standard neurosurgical procedures that have been described elsewhere $(34,37)$. Briefly, after the scalp was prepared with an antiseptic, a supratentorial occipital right paramedial craniotomy centered over the desired location of array implantation was performed. After opening the dura toward the midline, the surface of the brain was exposed and the UEA was inserted using a pneumatic inserter (Blackrock Microsystems). The electrode's external connector was attached to the skull using six 5-mm titanium microscrews. The final array location was documented with intraoperative photographs and a postoperative computed tomography (CT) scan that was fused with the preoperative planning on 3D MRI.

After 3 months, the microscrews securing the external connector to the skull loosened, causing a minor displacement of the connector relative to the skull. The connector was remounted using new microscrews under local anesthesia. After 6 months, the subject was reanesthetized and a craniotomy was performed in order to explant the UEA. There was negligible fibrosis around the implanted UEA, and the UEA and the external connector were easily explanted without complications. The wound was closed in a standard way using subcutaneous reabsorbable sutures and stainless skin staples. The subject has been followed periodically after the explantation and reports no complications.

Impedance measurements. Prior to each stimulating and recording session, we performed an impedance test of all 96 electrodes at $1 \mathrm{kHz}$ using the "test electrodes" function of the CereStim 96 multichannel microstimulation system (Blackrock Microsystems). Impedance measurements were analyzed off line using custom MatLab software (MathWorks) and Python software (Python Language Reference v2.7 available at http://www.python.org).

Neural recording and stimulation. Electrical stimulation and multiunit neural recording sessions were generally performed 5 days per week (Monday to Friday), 1 to 2 times per day (morning and afternoon sessions) for up to 4 hours per session over a period of 6 months. Neural signals were recorded using the NeuroPort data acquisition system (Blackrock Microsystems). Constant current stimuli were delivered using the CereStim 96 and customized software was used to deliver multiple stimulation patterns to different subsets of electrodes, thereby creating custom stimulation configurations using interleaved channels. The electrical stimuli used in these experiments were delivered between the selected electrode (or a selected group of electrodes) and a deinsulated platinum wire that was positioned under the dura, near the implanted UEA. Because of limitations in the CereStim 96 stimulator, only 16 electrodes could be stimulated simultaneously. In some experiments, we also simultaneously recorded EEG signals at a sampling rate of $1000 \mathrm{~Hz}$ with the help of the Curry 7 platform (Compumedics). The number of hours of stimulation over the 6-month period was approximately 540 hours.

For removing stimulation artifacts, we used a fast independent component analysis (ICA) algorithm (53).

Optimization of the stimulation parameters. We determined psychometric curves for the detection and discrimination tasks. Each stimulation was accompanied by a "stimulating-now" tone. Different test stimulus amplitudes, ranging from 1 to $128 \mu \mathrm{A}$, were presented randomly until 10 responses for each test stimulus amplitude were recorded. In addition, $20 \%$ of the trials were randomized sham stimuli (no stimulus). Psychometric data were fit using a Weibull cumulative distribution function to estimate response thresholds (amplitude value at $50 \%$ probability of detection). The calculations were performed using the scipy.optimize function in the Python package SciPy (54).

The geometrical surface area of electrode tips was calculated from SEM micrographs of the explanted UEA by assuming conically shaped tips.

Threshold testing. A binary search procedure was used to measure phosphene thresholds on each of the 96 electrodes. Stimulation spanned 1 to $128 \mu \mathrm{A}$ with a minimum step size of $1 \mu \mathrm{A}$. In addition, we used $10 \%$ catch trials in which a null stimulus was presented. This technique allowed us to estimate the lowest stimulus intensity that produced reliable phosphene perceptions for each electrode. Briefly, we first randomly selected one UEA electrode and then we started stimulation using a binary convergence search. Each intensity was tested at least twice and we instructed the subject to consider only clear phosphenes. We started with an amplitude of $1 \mu \mathrm{A}$. If the subject did not perceive the stimulus, we increased the amplitude of the stimulus by a factor of 2 . If the subject perceived the new intensity, we performed a half-interval search between this value and the last unperceived amplitude until the volunteer was able to correctly identify the stimulus in 2 consecutive trials. This procedure was repeated until the subject was able to perceive 2 successive phosphenes evoked by a given stimulus amplitude or when we reached a maximum stimulation current of $128 \mu \mathrm{A}$. We then moved to the next randomly chosen electrode. This protocol allowed us to test all 96 electrodes in approximately 20 to 30 minutes and was used to periodically measure thresholds for all electrodes.

Accommodation of phosphenes to repeated stimulation. A stimulus of twice the threshold current level was presented 30 consecutive times. For these experiments, we used 50 charge-balanced cathodic first biphasic pulses, with several different pulse widths (100, 170, 400, and $800 \mu \mathrm{s})$ at a frequency of $300 \mathrm{~Hz}$, and adjusted the interpulse interval to keep the complete duration of each train to $166 \mathrm{~ms}$. Stimulation trains were presented every 4 seconds, which allowed sufficient time for the subject to respond verbally regarding the features of the perceptions and to perform a keyboard button press. Five electrodes were tested using this paradigm.

Two-point discrimination. A number of stimulation experiments were conducted with different pairs of electrodes distributed over the whole UEA. We also stimulated electrode pairs with a delay between stimuli to identify the minimum time interval that facilitated the perception of 2 discrete phosphenes. For these experiments, we used delays of 50, 100, 200, 250, 300, 400, 500, 700, 1000, 2000, and $4000 \mathrm{~ms}$ between the 2 trains of stimulating pulses.

Phosphene mapping. For mapping the location of phosphenes evoked by each microelectrode, we used our standard stimulus protocol. The subject was instructed to concentrate on holding her eye position as if she were looking straight ahead. Each stimulus was accompanied with a brief "stimulating-now" tone. If she perceived a phosphene, then she was requested to indicate the location of the phosphene with respect to the center of her visual field. To help with this task, we used a wireless drawing tablet (Wacom Intuos $\mathrm{M}$, Wacom Co. Ltd) on which we added a small post at the center of the tablet for tactile reference and orientation. In addition, we asked her 
to describe the qualities of her perceptions with respect to the shape, size, color, brightness (subjective ratings from 0 to 5), and possible motion of the evoked perceptions. The digitizing tablet was also used by the subject for drawing the perceptions elicited by the stimulation of multiple electrodes.

In some of these experiments, we used a portable eye tracking system (Tobii Pro Glasses 2) to measure the subject's eye movements. Our results showed that the phosphenes moved along with eye movements, which complicated the generation of consistent phosphene maps. To avoid this complication, before each electrical stimulation the participant placed her finger on the post and was asked to concentrate on holding her gaze straight at the center of her visual space.

Bio-inspired retinal encoder. The visual encoder system has been described elsewhere $\left(12,13,55^{-59}\right)$. In brief, it consisted of a video camera attached to an eyeglass frame for image acquisition using head scanning, and custom hardware/software that performs a real-time analysis of the light patterns that are received by the light sensors in the camera and a multichannel spatiotemporal filtering of the visual information to extract and enhance the most relevant features of the scene. The system also includes an additional stage that converts the output of the bio-inspired retinal encoder into stimulus parameters and reencodes this information into a neuromorphic stream of electrode addresses and stimulation amplitudes. The filtering in the retinal encoder was adjusted to change the pulse train amplitude sent to the electrodes to modulate phosphene brightness. Although the retinal encoder has the capability of communicating with hundreds of electrodes, the CereStim 96 electrode stimulator used in these experiments limited the delivery of simultaneous stimulation to 16 electrodes. These 16 electrodes were selected to stimulate via electrodes distributed over the entire UEA.

Training sessions. Training sessions lasted 10 to 15 minutes. Usually we performed several trials (20-30 stimulations/condition). The number of training sessions was dependent on the task to learn but as a rule we performed 4 to 6 training sessions per task.

Statistics. Statistical analysis was performed using IBM SPSS 20.0 statistics software. We used linear regression to determine significant changes in electrode thresholds over time and for investigating accommodation to repeated stimuli. Unless otherwise noted, data are reported as mean \pm standard deviation (SD) and averages were compared using $t$ test or ANOVA with Scheffe's procedure for multiple comparisons. In all cases, a $P$ value of less than 0.05 was considered statistically significant.

Study approval. The human experimentation was performed under a protocol that was approved by the Hospital General Universitario de Elche Clinical Research Committee and registered at ClinicalTrials.gov (NCT02983370). We followed all relevant ethical guidelines related to clinical trials regulation (EU no. 536/2014, repealing Directive 2001/20/EC), the Declaration of Helsinki, and the EU Commission Directives (2005/28/EC and 2003/94/EC), and we obtained written informed consent before any study procedure was conducted. All the procedures and risks were fully explained to the subject prior to her participation, emphasizing the investigational nature of the study and that she should not expect any short or long-term benefit resulting from her participation in the study. She understood that the main purpose of the study was to gain knowledge essential for the future development of a cortical visual neuroprosthesis for the blind.

\section{Author contributions}

EF and RAN designed the study. AA, PGL, PRR, and EF designed, collected, and interpreted preoperative and postoperative clinical data. PGL developed and performed the surgical implantation procedures. EF, AA, CSS, AML, SP, MDG, and AR performed all intracortical microstimulation experiments. CSS, TSD, EF, and RAN analyzed data from recording experiments. AR, SP, EF, and RAN analyzed data from impedance measurements. EF, AA, CSS, PGL, AML, SP, MDG, AR, BG, XC, PRR, JDR, TSD, and RAN analyzed data from intracortical microstimulation experiments. All authors contributed to the interpretation of the results. EF and RAN completed the initial draft of the manuscript, and all authors provided critical review, edits, and approval for the final manuscript.

\section{Acknowledgments}

We would like to thank the study subject (BG) and her husband for their extraordinary commitment to this study and their patience with experiments. We are very grateful to all the people from IMED Hospital Elche, especially to Carlos Yago, Enrique Ibañez, and Angel Gomez for all their help and support and for providing clinical care and monitoring throughout the study. We would also like to thank Joaquín López (Bidons Egara Research Chair) and Randall J. Olson (John A. Moran Eye Center, University of Utah) for their invaluable help and support during the planning and development of this research, and Noah C. Benson (University of Washington) and Jennifer Sorinas (University of Zurich) for all their help with the retinotopic analysis of the MRI images. Funding was provided by grant RTI2018-098969-B-100 from the Spanish Ministerio de Ciencia Innovación y Universidades, by grant PROMETEO/2019/119 from the Generalitat Valenciana, by the Bidons Egara Research Chair of the University Miguel Hernández, by the John A. Moran Eye Center of the University of Utah, by a career development award from the NIH National Institute for Neurological Disorders and Stroke (K23 NS114178), and by an unrestricted grant from Research to Prevent Blindness to the Department of Ophthalmology and Visual Sciences, University of Utah..

Address correspondence to: Eduardo Fernández, Universidad Miguel Hernández, Instituto de Bioingeniería, Avda de la Universidad, s/n. 03202 Elche, Spain. Phone: 34.965222001; Email: e.fernandez@umh.es.
1. Brindley GS. Effects of electrical stimulation of the visual cortex. Hum Neurobiol. 1982;1(4):281-283.

2. Brindley GS, Lewin WS. The sensations produced by electrical stimulation of the visual cortex. JPhysiol. 1968;196(2):479-493.

3. Brindley GS, Lewin WS. Short and long-term stability of cortical electrical phosphenes.
JPhysiol. 1968;196(2):479-493.

4. Dobelle WH, Mladejovsky MG. Phosphenes produced by electrical stimulation of human occipital cortex, and their application to the development of a prosthesis for the blind. JPhysiol. 1974;243(2):553-576.

5. Dobelle WH, et al. 'Braille' reading by a blind volunteer by visual cortex stimulation. Nature. 1976;259:111-112.

6. Dobelle WH, et al. Artificial vision for the blind: electrical stimulation of visual cortex offers hope for a functional prosthesis. Science. 1974;183:440-444.

7. Beauchamp MS, et al. Dynamic stimulation of 
visual cortex produces form vision in sighted and blind humans. Cell. 2020;181(4):774-783.e5.

8. Roelfsema PR. Writing to the mind's eye of the blind. Cell. 2020;181(4):758-759.

9. Fernandez E, et al. Toward long-term communication with the brain in the blind by intracortical stimulation: challenges and future prospects. Front Neurosci. 2020;14:681.

10. Schmidt EM, et al. Feasibility of a visual prosthesis for the blind based on intracortical microstimulation of the visual cortex. Brain.1996;119(pt 2):507-522.

11. Normann RA, Fernandez E. Clinical applications of penetrating neural interfaces and Utah Electrode Array technologies. J Neural Eng. 2016;13(6):061003.

12. Normann RA, et al. Toward the development of a cortically based visual neuroprosthesis. J Neural Eng. 2009;6(3):035001.

13. Fernandez E, et al. Development of a cortical visual neuroprosthesis for the blind: the relevance of neuroplasticity. J Neural Eng. 2005;2(4):R1-12.

14. Chen X, et al. Shape perception via a high-channel-count neuroprosthesis in monkey visual cortex. Science. 2020;370(6521):1191-1196.

15. Normann RA. Technology insight: future neuroprosthetic therapies for disorders of the nervous system. Nat Clin Pract Neurol. 2007;3(8):444-452.

16. Negi S, et al. In vitro comparison of sputtered iridium oxide and platinum-coated neural implantable microelectrode arrays. Biomed Mater. 2010;5(1):15007.

17. Bittner AK, et al. A survey of photopsias in self-reported retinitis pigmentosa: location of photopsias is related to disease severity. Retina. 2009;29(10):1513-1521.

18. Bittner AK, et al. Photopsias are related in part to perceived stress and positive mood in retinitis pigmentosa. Eye (Lond). 2012;26(1):101-108.

19. Boller F, et al. Charles bonnet syndrome and other hallucinatory phenomena. Front Neurol Neurosci. 2018;41:117-124.

20. Cogan SF, et al. Tissue damage thresholds during therapeutic electrical stimulation. JNeural Eng. 2016;13(2):021001.

21. Shannon RV. A model of safe levels for electrical stimulation. IEEE Trans Biomed Eng. 1992;39(4):424-426.

22. Benson NC, et al. The retinotopic organization of striate cortex is well predicted by surface topology. Curr Biol. 2012;22(21):2081-2085.

23. Butt $\mathrm{OH}$, et al. The fine-scale functional correlation of striate cortex in sighted and blind people. JNeurosci. 2013;33(41):16209-16219.

24. Rushton DN, Brindley GS. Properties of cortical electrical phosphenes. In: Cool SJ, Smith EL, eds. Frontiers in Visual Science. Springer-Verlag; 1978:574-593.

25. Flesher SN, et al. Intracortical microstimulation of human somatosensory cortex. Sci Transl Med. 2016;8(361):361ra141.

26. Aflalo T, et al. Neurophysiology. Decoding motor imagery from the posterior parietal cortex of a tetraplegic human. Science. 2015;348(6237):906-910

27. Bouton CE, et al. Restoring cortical control of functional movement in a human with quadriplegia. Nature. 2016;533(7602):247-250.

28. George JA, et al. Long-term performance of Utah slanted electrode arrays and intramuscular electromyographic leads implanted chronically in human arm nerves and muscles. J Neural Eng. 2020;17(5):056042.

29. Willett FR, et al. High-performance brain-totext communication via handwriting. Nature. 2021;593(7858):249-254.

30. Bullard AJ, et al. Estimating risk for future intracranial, fully implanted, modular neuroprosthetic systems: a systematic review of hardware complications in clinical deep brain stimulation and experimental human intracortical arrays. Neuromodulation. 2020;23(4):411-426.

31. Simeral JD, et al. Home use of a percutaneous wireless intracortical brain-computer interface by individuals with tetraplegia. IEEE Trans Biomed Eng. 2021;68(7):2313-2325.

32. Marin C, Fernandez E. Biocompatibility of intracortical microelectrodes: current status and future prospects. Front Neuroeng. 2010;3:8.

33. Fernandez E, Botella C. Biotolerability of intracortical microelectrodes. Advanced Biosystems. 2017;2(1):1-14.

34. Fernandez E. Development of visual neuroprostheses: trends and challenges. Bioelectron Med. 2018;4(8):1-8.

35. Warren DJ, et al. High-resolution two-dimensional spatial mapping of cat striate cortex using a 100-microelectrode array. Neuroscience. 2001;105(1):19-31.

36. Wilms M, et al. Comparison of functional and cytoarchitectonic maps of human visual areas $\mathrm{V} 1, \mathrm{~V} 2, \mathrm{~V} 3 \mathrm{~d}, \mathrm{~V} 3 \mathrm{v}$, and $\mathrm{V} 4(\mathrm{v})$. Neuroimage. 2010;49(2):1171-1179.

37. Fernandez E, Normann RA. CORTIVIS approach for an intracortical visual prostheses. In: Gabel VP, ed. Artificial Vision. Springer International Publishing; 2017:191-201.

38. Niketeghad S, et al. Phosphene perceptions and safety of chronic visual cortex stimulation in a blind subject. J Neurosurg. 2019;132(6):2000-2007.

39. Bosking WH, et al. Saturation in phosphene size with increasing current levels delivered to human visual cortex. J Neurosci. 2017;37(30):7188-7197.

40. Self MW, et al. The segmentation of proto-objects in the monkey primary visual cortex. Curr Biol. 2019;29(6):1019-1029.

41. Bak M, et al. Visual sensations produced by intracortical microstimulation of the human occipital cortex. Med Biol Eng Comput. 1990;28(3):257-259.

42. Winawer J, Parvizi J. Linking electrical stimulation of human primary visual cortex, size of affected cortical area, neuronal responses, and subjective experience. Neuron. 2016;92(6):1213-1219.

43. Troyk P, et al. A model for intracortical visual prosthesis research. ArtifOrgans. 2003;27(11):1005-1015.

44. Cha K, et al. Simulation of a phosphene-based visual field: visual acuity in a pixelized vision system. Ann Biomed Eng. 1992;20(4):439-449.

45. Dagnelie G, et al. Paragraph text reading using a pixelized prosthetic vision simulator: parameter dependence and task learning in free-viewing conditions. Invest Ophthalmol Vis Sci. 2006;47(3):1241-1250.

46. Klink PC, et al. Distinct feedforward and feedback effects of microstimulation in visual cortex reveal neural mechanisms of texture segregation. Neuron. 2017;95(1):209-220.

47. McCreery DB, et al. Stimulus parameters affecting tissue injury during microstimulation in the cochlear nucleus of the cat. Hear Res. 1994;77(1-2):105-115.

48. McCreery D, et al. The effects of prolonged intracortical microstimulation on the excitability of pyramidal tract neurons in the cat. Ann Biomed Eng. 2002;30(1):107-119.

49. Pancrazio JJ, et al. Thinking small: progress on microscale neurostimulation technology. Neuromodulation. 2017;20(8):745-752.

50. Kim S, et al. Integrated wireless neural interface based on the Utah electrode array. Biomed Microdevices. 2009;11(2):453-466.

51. Rush AD, Troyk PR. A power and data link for a wireless-implanted neural recording system. IEEE Trans Biomed Eng. 2012;59(11):3255-3262.

52. Fernandez E, et al. Mapping of the human visual cortex using image-guided transcranial magnetic stimulation. Brain Res Brain Res Protoc. 2002;10(2):115-124.

53. Lu Y, et al. Using independent component analysis to remove artifacts in visual cortex responses elicited by electrical stimulation of the optic nerve. J Neural Eng. 2012;9(2):026002.

54. Virtanen P, et al. SciPy 1.0: fundamental algorithms for scientific computing in Python. Nat Methods. 2020;17:261-272.

55. Morillas CA, et al. A design framework to model retinas. Biosystems. 2007;87(2-3):156-163.

56. Pelayo FJ, et al. Traslating image sequences into spike patterns for cortical neuro-stimulation. Neurocomputing. 2004;58(60):885-892.

57. Lozano A, et al. A 3D convolutional neural network to model retinal ganglion cell's responses to light patterns in mice. Int J Neural Syst. 2018;28(10):1850043.

58. Martinez-Alvarez A, et al. Automatic tuning of a retina model for a cortical visual neuroprosthesis using a multi-objective optimization genetic algorithm. Int J Neural Syst. 2016;26(7):1650021.

59. Lozano A, et al. Neurolight: a deep learning neural interface for cortical visual prostheses. Int $J$ Neural Syst. 2020;30(9):2050045. 